Aus Hofrath Dr. A. Theilhaber's Frauenheilanstalt in München.

\title{
Zur Pathologie und Therapie des sogenannten Mittelschmerzes.
}

Von

\section{A. Theilhaber.}

Der Lehre von dem Mittelschmerz oder, wie man ihn wobl richtiger bezeichnen dürfte, von der Intermenstruationskolik wurde bisher, wie mir scheint, nicht so viel Beachtung geschenkt, als sie verdient. Auch gehen die Ansichten über deren Entstehung und Behandlung recht weit aus einander. Die letztere war nach der Beschreibung der meisten Autoren bisher von sehr geringen Erfolgen begleitet. Es dürfte wohl am Platze sein, dieses Thema wieder einmal $\mathrm{zu}$ besprechen.

Was die Frequenz dieser Erkrankung betrifft, so gilt sie bei den meisten Aerzten als ein ziemlich seltenes Vorkommniss. Diese Auffassung hatte ich auch lange Zeit. Nach Beobachtungen der letzten Jahre scheint sie mir jedoch irrig zu sein. Dieser Irrthum ist offenbar dadurch entstanden, dass die meisten Frauen die Periodicität dieser Erscheinung nicht beachten. Am meisten ist dies natürlicher Weise in den niederen Ständen der Fall. Dem entsprechend sind in meiner poliklinischen Praxis in vielen Jahren Fälle von Intermenstrualkolik nicht diagnosticirt worden. Vor einigen Monaten gab ich meinem Assistenten in der Poliklinik, der dort alle Anamnesen aufnimmt, eine längere Anleitung diesbezüglich, schärfte $\mathrm{ihm}$ jedoch streng ein, nichts in die Patientinnen hineinzuexaminiren. Der Erfolg war, dass er in relativ kurzer Zeit 3 Fälle von intermenstrueller Kolik in der Poliklinik entdeckte.

Will man einen Ueberblick über die Häufigkeit der Erkrankung haben, so muss man in allen Fällen von zeitweise auftretenden 
Schmerzen, Blutungen und Ausfluss die Patientinnen veranlassen, genaue Notizen über das Datum der Menstruation und das Datum der betreffenden Krankheitssymptome zu machen. Nachdem ich es in dieser Weise arrangirt hatte, habe ich nun im Zeitraum eines Jahres allein 12 derartige Fälle entdeckt, während ich früher durchschnittlich alle ein bis zwei Jahre einmal einen Fall in der Privatpraxis constatirt hatte.

Die intermenstruellen Beschwerden zeichnen sich dadurch aus, dass regelmässig genau in der Mitte zwischen zwei Menstruationen sich entweder Schmerz oder Blutung oder Ausfluss einstellen. Jedes dieser drei Symptome kann allein auftreten, es können jedoch auch zwei oder drei dieser Symptome combinirt vorkommen. Am seltensten scheint die intermenstruelle Blutung zu sein, am häufigsten der intermenstruelle Ausfluss. Zur Kenntniss des Arztes kommen am häufigsten die Fälle mit intermenstruellen Schmerzen, da natürlich ein mehrtägiger Schmerz viel häufiger die Frauen veranlasst, zum Arzt zu gehen, als ein einige Tage dauernder Ausfluss. Der Schmerz ist von verschieden langer Dauer. Manchmal dauert er nur wenige Stunden, manchmal jedoch zwei bis drei Tage. Er wird übereinstimmend als krampfartig gesehildert. Er ist häufig mit Dysmenorrhoe (Menstrualkolik) vergesellschaftet. Die Patientinnen, die diese beiden Störungen haben, pflegen anzugeben, dass der Schmerz der beiden ausserordentlich ähnlich sei. Meist wird jedoch der Schmerz bei der echten Menstruation als heftiger geschildert, zuweilen ist jedoch auch das Umgekehrte der Fall. Der Schmerz hält gewöhnlich nicht ununterbrochen an, sondern macht Pausen von mehreren Minuten, oft von einer halben Stunde, einer Stunde und länger. Manche Patientinnen geben an, dass am Ende des Schmerzanfalles Ausfluss sich bemerkbar macht und dass jedesmal mit dem Fühlbarwerden des Ausflusses auch die Schmerzanfälle pausiren.

Der Ausfluss ist zuweilen sehr stark. Er dauert meist 2 bis 3 Tage und ist meist hell schleimig.

Wo Blutung vorhanden ist, pflegt dieselbe geringgradig zu sein, oft nur wenige Stunden, manchmal jedoch 2-3 Tage lang dauernd. Häufig ist vor dem Eintritt und nach Beendigung der Blutung noch ein einen Tag dauernder Ausfluss vorhanden.

Ueber die Ursachen dieser Anomalie gehen die Ansichten weit aus einander. Schäffer (Veit's Handbuch für Gynäkologie 1908) sagt: „Ueber die Entstehung des Mittelschmerzes wissen wir 
556 Theilhaber, Pathologie u. Therapie des sogenannten Mittelschmerzes.

so gut wie nichts. - Auch die Literatur geht recht kurz über diesen Punkt hinfort. Die Erklärungsversuche van der Velde's (flacheres und tieferes Wellenthal) helfen über die Schwierigkeiten kaum hinweg. Dass der Mittelschmerz wie die Menstruation mit der Ovarialfunction in einem inneren Zusammenhang steht, geht freilich aus seiner Regelmässigkeit und Periodicität hervor." B. Schultze (Centralblatt für Gynäkologie 1889, S. 331) meint diesbezüglich: "Nicht nur die Entleerung des Eiters, auch die Eiterabsonderung ist in manchen Fällen periodisch, häufig begleitet von einer mitten zwischen zwei Perioden auf einige Tage oder selbst Stunden beschränkten Eiterabsonderung aus dem Uterus. Die Beobachtung mit dem Probetampon weist das nach."

Hofmeịer '(Handbuch für Frauenkrankheiten 1908) schreibt, dass diese Schmerzanfälle nicht immer von krankhaften Processen am Uterus herrühren. Fasbender (Zeitschrift für Geburtshülfe und Frauenkrankheiten, 1. Band; 1. Heft) hält es für wahrscheinlich, dass bei Frauen mit krankhaft gesteigerter Erregbarkeit des ganzen Nervensystems oder der Eierstocknerven bei pathologischen Zuständen dieser Organe der Reiz durch physiologische Vorgänge in den Ovarien sich schon in kürzerer Zeit als den normalen 4 Wochen (nicht immer genau in 14 Tagen) hinreichend summirt hätte, um einen Reflexausschlag in Form einer Congestion nach den Genitalien herbeizuführen. In der sich an den Fasbender'schen Vortrag anschliessenden Discussion erklärte Ebell (Ebenda), dass er die Ursache dieser Schmerzen nicht in den Ovarien, sondern in der Anteflexion des Uterus sehe, der sich bestrebe, retinirte Flüssigkeiten (Fremdkörper) auszustossen.

Jacquet (Ebenda) hat in den von ihm beobachteten Fällen von Mittelschmerz stets chronische Beckenperitonitis beobachtet und hält die Annahme entzündlicher Vorgänge in den Ovarien und dadurch bedingte Beeinträchtigung des wachsenden Follikels für die plausibelste Erklärung.

E. Martin meinte in jener Discussion, es möchten in einzelnen Fällen Erkrankungen der Ovarien, in andern eine abnom gesteigerte Erregbarkeit des Nervensystems zu beschuldigen sein. Fehling (Dieses Archiv, Band 17, Heft 2) sagt: "Gemeinsam ist ferner, dass bei allen Patientinnen Störungen in den Sexualorganen vorhanden waren, und zwar wesentlich als chronisch entzündlicher Vorgang in der Umgebung des Uterus und der Ovarien." 
Theilhaber, Pathologie u. Therapie des sogenannten Mittelschmerzes. 557

Beigel (Die Krankheiten des weiblichen Geschlechts. 1863. S. 333) erwähnt die "Dysmenorrhoea intermenstrualis", glaubt aber nicht, dass dieses Krankheitsbild diesen Namen verdiene. Er meint, dass die schmerzhaften Symptome nicht mit der Menstruation in Verbindung gebracht werden können, sondern dass irgend eine Ent. zündung der Genitalien oder eine Neurose Schmerzempfindungen macht, die gar keinen Zusammenhang mit den menstrualen Vorgängen hätten. Karl Schröder (Lehrbuch der Gynäkologie) sagt, die Ursache dieser typisch wiederkehrenden Schmerzen sei nicht ganz klar; in allen wohl charakterisirten Fällen, die er beobachtet hat, finden sich eklatante Erkrankungen des Genitalapparates, so in einem besonders typischen Fall eine fixirte Retroflexio. Palmer (Amer. journ. of obst. Vol. XXXI. p. 328) berichtet über 6 Fälle von Mittelschmerz, die er in den letzten 12 Jahren beobachtet hat. Er sucht die Ursache in einer Oophoritis oder Perioophoritis, die irgend ein Hinderniss bei der Entleerung des Graaf'sehen Follikels bildet.

Addinsell (Transact. of the the obst. soc. London, 2. März 1898) sagt: In 4 Fällen von Mittelschmerz fand er Tubenschwellungen vor. Er glaubt, dass der Tubenschmerz hervorgerufen wird durch Zusammenziehungen der Tuben, die bestrebt sind, ibren Inbalt auszustossen.

Van der Velde versucht eine Erklärung durch den wellenförmigen Verlauf der Lebensvorgänge der Frau. Er unterscheidet Typen mit flacherem und tieferem Wellenthal. Bei ungewöhnlichem tiefen Wellenthal entsteht der Mittelschmerz, wenn die Wellenlinie ihren tiefsten Punkt erreicht hat.

Rosner (Centralbl. f. Gyn. 1906, S. 38) vertritt bezüglich der Entstehung des Leidens die Ansicht, dass nur bei pathologischen Veränderungen der Genitalorgane Mittelschmerz vorkommen kann. Er neigt der Ansicht von Bouilly zo, dass der Mittelschmerz durch chronische Congestionen der inneren Genitalorgane, welche durch vasomotorische, vom Eierstock ausgehende Störungen hervorgerufen worden sind, erzeugt wird. Auf Grund eigener Erfahrungen bestätigt er Richelot, dass die Congestionen durch diffuse pathologische Hypertrophie des Uterus zusammen mit Sklerose und kleincystischer Degeneration der Ovarien verursacht würden.

Brodier (Congrès international de Gyn., Amsterdam 1899) meint, die Ursachen des Mittelschmerzes seien entweder eine nervöse 
558 Theilhaber, Pathologie u. Therapie des sogenannten Mittelschmerzes.

Ueberreizung oder eine periodische Schwellung des antevertirten Uterus. -

Unter den 21 Fällen, die ich beobachtete, fanden sich zweimal Residuen beiderseitiger Salpingitis mit kleincystischer Degeneration der Ovarien. Die eine Patientin hatte ausser der dreitägigen Intermenstrualkolik und eines während dieser Zeit stattfindenden Ausflusses keinerlei Störung. In einem zweiten Falle bestanden ebenfalls Residuen einer beiderseitigen gonorrhoischen Salpingitis jedoch bei normalen Eierstöcken. Eine dritte Patientin hatte chronische Metritis und 6 kleine Adenome der Uterusschleimhaut. Im Vordergrunde standen bei dieser Patientin dreitägige Blutungen in der Mitte zwischen zwei Perioden. Bei den übrigen 18 Patientinnen war eine Abnormität an den Genitalien nicht nachweisbar. Bei den ersten beiden Fällen ist es mir nach dem weiteren Verlauf zweifelhaft geworden, ob die Tubenerkrankung die alleinige und vorwiegende Ursache der Schmerzen war. Sämmtliche Patientinnen waren nervös. Die Mehrzahl gab an, dass seit dem Bestehen des Mittelschmerzes auch die Nervosität schlimmer geworden sei.

Ich habe deshalb die Ansicht, dass pathologische Veränderungen der Genitalien durchaus nicht nothwendig für die Entstehung der Intermenstrualkolik sind, bezw. dass, wo sie vorhanden sind, sie häufig nur zufällige Complicationen vorstellen. Dass Congestivzustände zu den Geschlechtstheilen zu dieser Zeit vorhanden sind, wird durch das häufige Auftreten des Ausflusses und die zuweilen in die Erscheinung tretenden Blutungen höchst wahrscheinlich gemacht. Die strenge Periodicität der Schmerzen, ihr Auftreten genau in der Mitte zwischen zwei Perioden spricht für Abhängigkeit von der Function der Ovarien. Dass hiermit immer oder häufig das Platzen eines Follikels verbunden ist, wie manche Autoren meinen, ist sehr wahrscheinlich. Die Mehrzahl (16) meiner Patientinnen hatte nicht geboren, 14, wovon 13 Nullipare, klagten auch über Menstrualkolik.

Bezüglich der Behandlungsmethoden ist die Ausbeute in der bisherigen Literatur eine sehr dürftige. Hofmeier meint, es gäbe keine eigentliche Therapie, da es sich um einen physiologischen Process handle. Schultze empfiehlt häufige Ausspülung des Uterus. Fehling hat Besserung nach häufigen Scarificationen der Vaginalportion gesehen. In einem Falle, in der er die Castration anwandte, verschwanden die Beschwerden nicht vollständig. Van der Velde hat mit Erfolg Ovarintabletten gegeben. 
Da meine Patientinnen meist nervös waren, habe ich in früherer Zeit zunächst eine Behandlung des Nervensystems durch klimatische Curen, Hydrotherapie u.s.w. eingeleitet. Es wurde häufig Besserung, aber keineswegs Heilung erzielt. Ich habe dann Scarificationen in den Tagen, bevor die Schmerzen erwartet wurden, angewandt. Dies brachte vorübergehend Besserung. Bei der einen Patientin mit Verdickung der beiden Tuben und kleincystischer Degeneration der Ovarien exstirpirte ich die Tuben und beide Eierstöcke. Die Patientin ist seit 8 Jahren geheilt. Bei der anderen Patientin mit Verdickung beider Tuben und normalen Ovarien exstirpirte ich nur die beiden Tuben. Da die Patientin Werth auf die Erhaltung der Eierstöcke legte, entfernte ich dieselben nicht, in der irrigen Meinung, dass die Entfernung der kranken Tuben allein genügen würde, die Symptome zu beseitigen. Es war dies jedoch eine Täuschung, denn die Schmerzen blieben nach dieser Operation unverändert bestehen. Wahrscheinlich hatte bei der anderen Patientin die Entfernung der Ovarien, nicht die der Tuben, die Heilung bewirkt. Bei der Patientin, die intermenstrual blutete und Adenome besass, wurden natürlich letztere entfernt und die Blutungen hierdurch beseitigt.

Da ich die Beobachtung gemacht hatte, dass manche Congestivzustände der Genitalien auf eine Reihe von Monaten schwinden, wenn ein Aderlass vorgenommen wird, so habe ich bei drei Patientinnen Aderlässe angewandt. Es trat eine wesentliche Verringerung der Schmerzen auf. Doch kehrten die Schmerzen in verringerter Intensität nach 6-12 Monaten wieder, sodass dann eine Wiederholung des Aderlasses nothwendig wurde, der auch dann wieder eine beträchtliche Besserung herbeiführte. In den letzten Jahren bin ich wenigstens bei Nulliparen zu einer anderen Behandlung übergegangen, die mir recht befriedigende Erlolge gegeben hat. Ich habe bereits früher (Münchener med. Wochenschr., 1901, No. 22 und 23) auseinandergesetzt, dass ich die häufigste Ursache der Menstrualkolik in einer spastischen Contraction der Uterusmusculatur sehe, und zwar meist der ringförmigen Muskeln, die in der Gegend des inneren Muttermundes sich finden und dort eine Art Sphineter bilden. Dass ein solcher ringförmiger Muskel existirt, wurde neuerdings auch wieder durch die Untersuchungen von v. Franqué bestätigt. Früher hat schon Sappey (Traité d'anatomie descriptive, Paris 1873) diesen Ringmuskel beschrieben. Auch in Franks Lehrbuch der Anatomie der Hausthiere findet sich folgender Satz: 
560 Theilhaber, Pathologie u. Therapie des sogenannten Mittelschmerzes.

"Die Kreisfasern bilden am Halse des Uterús eine 15-17 mm starke Schicht, die als organischer Schliessmuskel bezeichnet wird. Den inneren Theil dieser Muskelschicht deutet Ellenberger als. Muscularis mucosae." Contractionen dieser Ringmusculatur kann man vor Allem bei Krampfwehen in der Eröffnungsperiode der Geburt beobachten. - Wenn man behufs intrauteriner Behandlung eine mit. Watte umwickelte Sonde in ein starkes Aetzmittel taucht und sehr langsam durch die Cervix führt, so kommt man meist ungehindert bis zum inneren Muttermund, verweilt man hier einige Secunden, so tritt zuweilen eine starke Verengerung auf, die sogar manchmal der feinsten Sonde den Durchtritt verweigert. Es kann die Verengerung so hochgradig werden, dass sie dem völligen Verschluss des inneren Muttermundes gleichkommt. Will man die Sonde mit Sicherheit in das Cavum uteri bringen, so ist es deshalb Regel, die Gegend des inneren Muttermundes mit grösster Schnelligkeit zu passiren, ehe er Zeit hat, sich zu contrahiren.

Derartige Spasmen treten bei der Menstruation häufig auf, namentlich bei nervösen Patientinnen. Dieselben können häufig Schmerzen verursachen, auch wenn Blut nicht retinirt ist. Dieser Spasmus kann schon auftreten zu einer Zeit, wo noch gar kein Blut ausgeschieden ist. Es kann aber auch ab und zu sich während der Blutung durch den Spasmus am inneren Muttermund vorübergehend ein Verschluss ausbilden, so dass die Längsmusculatur des Uterus sich kräftig abmüben muss, um das retinirte Blut durch den inneren Muttermund durchzutreiben. Nothwendig für die Entstehung des Schmerzes ist jedoch die Retention des Blutes nicht. Wir wissen, dass krankhafte Contractionen auch sonst an muskelhaltigen Organen vorkommen und die heftigsten Kolikschmerzen verursachen. Solche Spasmen sind 7. B. bekannt unter dem Namen des Magenkrampfes, sie finden sich dann an Cardia und Pylorus. Beim Darmkrampf kommen derartige spastische Contractionen an verschiedenen Abschnitten des Dünn- und Dickdarmes vor, auch am Mastdarm werden solche Spasmen beobachtet, häufig mit, jedoch zuweilen auch ohne Fissuren am Anus. Im Oesophagus. sind Spasmen ohne anatomische Veränderungen beobachtet worden, durch die die Patientinnen unfähig gemacht wurden, Speise und Trank in den Magen zu bringen, so dass sogar feine Schlundsonden zeitweise nicht passiren konnten, während am nächsten Tage die stärksten Schlundsonden leicht in den Magen rutschten. 
Theilhaber, Pathologie u. Therapie des segenannten Mittelschmerzes. 561

Auch die Bleikolik erzeugt solche spastische Contractionen im Darm.

Diese Spasmen der Uterusmusculatur sind es meiner Ansicht auch, die die Intermenstrualkolik verursachen. Zu ihrer Beseitigung habe ich in neuester Zeit dieselbe Behandlung vorgenommen, die ich auch bei der Menstrualkolik angewandt habe. Bei letzterer pflegt eine Erweiterung des inneren Muttermundes auf einige Monate die Schmerzen zu beseitigen. Hierauf, auf die den intraüterinen Ausspülungen vorausgehende Erweiterung des inneren Muttermundes, führe ich auch den Erfolg der Schultzo'schen Methode zurück. Ein dauernder Erfolg scheint mir nach früheren Beobachtungen bei der Dysmenorrhoe durch die Schultze'sche Behandlung nur selten erreicht zu werden. Analog der Behandlung der Krämpfe am Anus habe ich deshalb bei der Behandlung der Dysmenorrhoe den Sphincter am inneren Muttermund ursprünglich durch Einschnitt in denselben geschwächt und dadurch die Krämpfe oft beseitigt. Später bin ich noch zu einem energischeren Verfahren übergegangen, indem ich kleine Stücke aus der Musculatur in der Gegend des inneren Muttermundes excidirte. Bezüglich der Erfolge haben mir eine Reihe von Collegen mitgetheilt, dass sie mit dem Errgebniss der Behandlunġsart bei der Menstrualkolik sehr zufrieden waren (WilleBraunschweig, Ludwig-Hirsch-Charlottenburg u. A.).

Ich selbst habe diese Resectio orificii interni in 12 Jahren an über 100 Fällen angewandt. Zwei Drittel der Kranken verloren ihre Beschwerden ganz oder nahezu ganz, bei $1 / 6$ wurden sie gebessert und verkürzt und bei einer kleinen Anzahl von Fällen (meist schwere Hystericae) versagte das Verfahren vollständig.

Ich habe nun diese Behandlung auch auf die Intermenstrualkolik der Nulliparen übertragen, in der Meinung, dass es sich auch hier um einen Spasmus der Musculatur am inneren Muttermund auf nervöser Basis handele und dass die Schwächung der Ringmusculatur diesen Krampf beseitigen werde.

In der That verloren von fünf Patientinnen drei die Kolik vollständig, bei einer besserte sich dieselbe wesentlich und bei einer hatte die Behandlung keinen Erfolg. In letzterem Falle gelang es dann mittels nochmaliger Dilatation, Aderlass von $300 \mathrm{ccm}$, Anwendung von $38^{\circ} \mathrm{C}$. warmen Sitzbädern (1 Stunde lang) und Verabreichung von Aspirintabletten während des Anfalles die Schmerzen auf ein Minimum zu reduciren. 
562 T'heilhaber, Pathologie u. Therapie des sogenannten Mittelschmerzes.

Was die Technik der Resectio orifieii interni betrifft, so pflege jch zunächst mittels Oberländer's Dilatatoren bis etwa $10 \mathrm{~mm}$ den inneren Muttermund zu dilatiren, dann mittels Schultze's. Dilatator zu spreizen, dann eine Discission zu machen. Jetzt gelingt es gewöhnlich leicht, mittels meines Dysmenorrhoemessers (bei Stiefenhofer, hier, käuflich) aus der vorderen und hinteren Muttermundslippe in der Gegend des Orificium internum uteri ein. Stück zu excidiren. Der Erfolg ist manchmal ein sofortiger, manchmal tritt er erst nach 2-3 Monaten (nach vollständiger Vernarbung: der Wunde) ein. 